\title{
Epidemiological, Clinical and Serological Characteristics of COVID-19 in Symptomatic Patients in Fkih Ben Salah Province (Morocco)
}

\author{
Hicham Esselmani ${ }^{1}$, Asmaa Yassine ${ }^{2}$, Boutaina Akhatar $^{3}$ and Abderrazak Lfakir ${ }^{4}$
}

${ }^{1}$ Department of Clinical Microbiology, HAFIR Medical Laboratory, Fkih Ben Salah, Morocco.

${ }^{2}$ Medicine service A, Regional Hospital Center, Beni Mellal, Morocco.

${ }^{3}$ Medical Laboratory, Provincial Hospital Center, Fkih Ben Salah, Morocco.

"Correspondence:

Hicham Esselmani, HAFIR Laboratory of Medical Analysis Lakouacem, street $28 \mathrm{n}^{\circ} 12$. Fkih Ben Salah, Morocco, Tel: +212662067101; Fax: +212523435724.

Received: 01 November 2021; Accepted: 22 December 2021

${ }^{4}$ Department of Physics, Faculty of Sciences and Techniques, University Moulay Slimane, Beni Mellal, Morocco.

Citation: Esselmani H, Yassine A, Akhatar B, et al. Epidemiological, Clinical and Serological Characteristics of COVID-19 in Symptomatic Patients in Fkih Ben Salah Province (Morocco). Trends Int Med. 2021; 1(2): 1-3.

At the time of writing this article, data on the clinical and epidemiological characteristics of COVID-19 contaminated Moroccan patients remains larlegy unavailable. Herein, we profiled retrospectively the epidemiological characteristics (clinical, serological) of 108 COVID-19 suspected cases in Fkih Ben Salah Province of Morocco.

Coronavirus disease 2019 (COVID-19) is caused by severe acute respiratory syndrome coronavirus 2 (SARS-CoV-2) [1]. Its manifestations can be viewed as a combination of the two processes, namely viral pneumonia and acute respiratory distress syndrome (ARDS) [2]. COVID-19 is a novel disease reported initially in Wuhan, Hubei Province, China, in December 2019 before it has become a global pandemic.

The Clinical signs of Covid-19 infection overlap with other viral infections, which has complicated the clinical diagnosis. Serological tests are considered powerful complements to the nucleic acid tests, especially for COVID-19 patients with undetectable viral RNA [3]. A clear advantage of serological tests over RT-PCR is the identification of individuals previously infected by SARS-CoV-2, even if they never underwent testing while acutely ill. As such, serological tests could be deployed as a surveillance tool to better understand the epidemiology of SARSCoV-2 and potentially inform individual risk of future disease [4].

This study aims to analyze the epidemiological, clinical and serological characteristics of patients with COVID-19 after diagnosis through detection of $\operatorname{IgM}$ and $\operatorname{IgG}$ antibodies against SARS-CoV-2 by fluorescence immunoassay. To address our objective, a survey was carried out on a total of 108 symptomatic patients who benefited from serological tests of covid-19 (November $1^{\text {st }}$ to December $31^{\text {st }}, 2020$ ) by a fluorescence immunoassay using the FREND System for the qualitative detection of anti-coronavirus $\operatorname{IgG}$ and $\operatorname{IgM}$ in human serum and plasma (Li-heparinized, EDTA, or citrate). Out of the total patients suspected with COVID-19 in the Province of Fkih Ben Salah, 103 patients were COVID-19 Positive.

Data in Table 1 shows patients age ranged from 18 to 90 years (mean age of 51.79 years) with 49 women and 59 men with a sex ratio of 1.2. Among the patients, almost none had severe SARSCoV-2 infections, except for two patients $(1.9 \%$ of patients with COVID-19) who were admitted to the hospital for severe acute respiratory syndrome. There was no difference in the proportion of men and women, which was inconsistent with the results of a study that was conducted by Guan et al. [5] that men were more likely to be infected than women. Similar result has been demonstrated by a Danish research team [6].

Underlying comorbidities were present in $11 \%(\mathrm{n}=12)$. Diabetes $(5.5 \%)$ and high blood pressure $(0.9 \%)$ were the most common comorbidities noted (Table 1). This value remains low as compared to the meta-analysis findings in China; where hypertension, cardiovascular disease, and diabetes $(16.4 \%, 12.1 \%$, and $9.8 \%$, respectively) [7] were the most common comorbidities noted. 
Table 1: Epidemic context and symptoms of the patients suspected with COVID-19 in the Province of Fkih Ben Salah.

\begin{tabular}{|c|c|c|c|}
\hline & $\begin{array}{l}\text { COVID-19 } \\
\mathrm{IgM}+, \mathrm{IgG}+\text { or both }+(\mathrm{n}=103)\end{array}$ & $\begin{array}{l}\text { Non COVID-19 } \\
\text { IgM- and IgG - } \\
(\mathrm{n}=05)\end{array}$ & $\begin{array}{l}\text { All patients } \\
(\mathrm{n}=108)\end{array}$ \\
\hline \multicolumn{4}{|l|}{ Demographic: } \\
\hline Age & $55.5(18-90)$ & $51(46-58)$ & $51.8(18-90)$ \\
\hline Male & $55(53 \%)$ & $4(80 \%)$ & $59(55 \%)$ \\
\hline Female & $48(47 \%)$ & $1(20 \%)$ & $49(45 \%)$ \\
\hline \multicolumn{4}{|c|}{ Co-morbidities and risk factors: } \\
\hline Age $>70$ years & $5(4.6 \%)$ & 0 & $5(4.6 \%)$ \\
\hline Diabetes & $6(5.5 \%)$ & 0 & $6(5.5 \%)$ \\
\hline Hight blood pressure & $1(0.9 \%)$ & 0 & $1(0.9 \%)$ \\
\hline Heart disease & 0 & 0 & 0 \\
\hline Tuberculosis & 0 & 0 & 0 \\
\hline \multicolumn{4}{|l|}{ Symptoms: } \\
\hline Fever $\geq 38^{\circ} \mathrm{C}$ & $22(20.3 \%)$ & $2(1.8 \%)$ & $24(22.1 \%)$ \\
\hline Cough & $10(9.2 \%)$ & $1(0.9 \%)$ & $11(10.1 \%)$ \\
\hline Asthenia & $5(4.6 \%)$ & 0 & $5(4.6 \%)$ \\
\hline Dyspnea & 0 & 0 & 0 \\
\hline Myalgia & $7(6.4 \%)$ & 0 & $7(6.4 \%)$ \\
\hline Diarrhea & 0 & 0 & 0 \\
\hline Abdominal pain & 0 & 0 & 0 \\
\hline Anosmia & $5(4.6 \%)$ & 0 & $5(4.6 \%)$ \\
\hline Dysgeusia & $5(4.6 \%)$ & 0 & $5(4.6 \%)$ \\
\hline
\end{tabular}

The most common symptoms reported by both groups included fever $(22.1 \%)$ and cough $(10.1 \%)$, followed by myalgia $(6.4 \%)$, asthenia, anosmia and dysgeusia (4.6\%) (Table 1). Patients may initially present diarrhea and nausea a few days prior to fever, suggesting that fever is dominant but not the initial symptom of infection. A small proportion of patients can have a headache or hemoptysis [6] or even be relatively asymptomatic [8, 9]. Our results showed a similar prevalence and the most common symptoms reported by both groups included fever $(22.1 \%)$ and cough $(10.1 \%)$, followed by myalgia $(6.4 \%)$.

Out of 108 patients suspected with COVID-19, 103 patients $(95.3 \%)$ generated SARS-CoV-2 specific antibody responses. The most common profile is IgM negative/IgG positive $(48.5 \%)$, followed by IgM positive/IgG positive (43.6\%) and IgM positive/ IgG negative (7.9\%). A proportion of $92.2 \%(95 / 103)$ of these patients were positive for IgG and $51.4 \%$ of them $(53 / 103)$ were positive for IgM. It is worth noting the reported low of isolated IgM antibody positivity in most of the studies. We can speculate that this could be a false negative due to low antibody concentrations or their short duration.

The study by Li et al. [10] on large samples collected from 397 RT-PCR confirmed COVID-19 patients and 128 negative patients from eight different clinical centers using a rapid lateral flow immunoassay searching IgM and IgG antibodies. The IgM-IgG combined assay showed better sensitivity compared to the single IgM or IgG test. In fact, among the COVID-19 patients it was found that $64.48 \%(256 / 397)$ had both $\operatorname{IgM}$ and $\operatorname{IgG}$ positive test results, while $18.13 \%$ (72/397) and $6.04 \%$ (24 of 397) had only IgM, and only IgG positive results, respectively [10].

\section{Conclusion}

This study is the first of its kind to be conducted among symptomatic patients with suspected SARS-CoV-2 infection in the Province of Fkih Ben Salah. It provides important information on the demographic, anthropometric, clinical and serological characteristics of these patients. Most cases present the common coronavirus symptoms (fever and cough) with IgG antibodies to SARS-CoV-2.

\section{References}

1. Hsih WH, Cheng MY, Ho MW, et al. Featuring COVID-19 cases via screening symptomatic patients with epidemiologic link during flu season in a medical center of central Taiwan. J Microbiol Immunol Infect. 2020; 53: 459-466.

2. Gibson PG, Qin L, Puah S. COVID-19 ARDS: clinical features and differences to usual preCOVID ARDS. Med J Aust. 2020.

3. Huang C, Wang Y, Li X, Ren L, et al. Clinical features of patients infected with 2019 novel coronavirus in Wuhan, China. Lancet. 2020; 395: 497-506.

4. Maria I, Arianna D, Francesca LG, et al. Serological assays for SARS-CoV-2 infectious disease: Benefits, limitations and perspectives. IMAJ 2020; 22: 203-210.

5. Guan WJ, Ni ZY, Hu Y, et al. Clinical characteristics of 2019 novel coronavirus infection in China. medRxiv preprint first posted online. 2020; 382: 1708-1720.

6. Kragholm K, Andersen MP, Gerds TA, et al. Association between male sex and outcomes of Coronavirus Disease 2019 (Covid-19) - a Danish nationwide, register-based study. N Engl J Med. 2020; 382: 1708-1720.

7. Zhang JJY, Lee KS, Ang LW, et al. Risk factors of severe disease 
and efficacy of treatment in patients infected with COVID-19: a systematic review, meta-analysis and meta-regression analysis. Clinical Infectious Diseases. 2020; 71: 2199-2206.

8. LU S, Lin J, Zhang Z, et al. Alert for non-respiratory symptoms of coronavirus sisease 2019 (COVID-19) patients in epidemic period: a case report of familial cluster with three asymptomatic COVID-19 patients. J Med Virol. 2021; 93: 518-521.
9. Chan JF, Yuan S, Kok KH, et al. A familial cluster of pneumonia associated with the 2019 novel coronavirus indicating personto-person transmission: a study of a family cluster. Lancet. 2020; 395: 514-523.

10. Li Z, Yi Y, Luo X, et al. Development and clinical application of a rapid IgM-IgG combined antibody test for SARS-CoV-2 infection diagnosis. J Med Virol. 2020; 92: 1518-1524. 